\title{
Prognósticos de Resultados de Futebol Utilizando Aprendizagem de Máquinas
}

\author{
Marcus Patrick Acioli da Silva* \\ José Maria Pires de Menezes Jr. ${ }^{* *}$ \\ * Curso de Engenharia Mecânica, Universidade Federal do Piauí, PI, \\ (e-mail: acioli@protonmail.com). \\ ** Curso de Engenharia Elétrica, Universidade Federal do Piaui, PI, \\ (e-mail: josemenezesjr@ufpi.edu.br)
}

\begin{abstract}
An analisys of performance and accuracy of Support Vector Machine model in prediction of football outcomes is proposed in this paper. The performance of the regularization parameter C, submitted to different kernels was analyzed. The characteristics were obtained after a process of normalization of the data, through an average of the last matches of the teams. The model built under the polynomial kernel of degree 2 presented the best results $(75,76 \%)$. In addition, the performance of the SVM model was evaluated in relation to other supervised learning algorithms. The accuracy of the model was also compared with others papers.

Resumo: Uma análise de desempenho e precisão de um modelo de Máquinas de Vetores de Suporte na predição de resultados de futebol é proposta neste trabalho, que analisa atuação do parâmetro de regularização $C$, submetido a diferentes kernels. As características foram obtidas após um processo de normalização dos dados, por meio de uma média das últimas partidas das equipes. O modelo construído sob o kernel polinomial de grau 2 apresentou os melhores resultados $(75,76 \%)$. Além disso, avalia o desempenho do modelo SVM em relação a outros algoritmos de aprendizado supervisionado. Realiza, também, a comparação da acurácia do modelo com outras pesquisas.
\end{abstract}

Keywords: Machine learning; Support Vector Machines; Football predicts; Multi-Layer Perceptron; KNeighbors;

Palavras-chave: Aprendizagem de Máquinas; Máquinas de Vetores de Suporte; Prognósticos de Futebol; Perceptron multicamadas; KNeighbors;

\section{INTRODUÇÃO}

A previsão de resultados de partidas de futebol sempre despertou o interesse dos pesquisadores ao longo do tempo, principalmente após o surgimento de casas de apostas e ainda mais, após a migração dessas para o ambiente virtual. Em seu relatório anual Betfair (2018) aponta um lucro, em 2017 de 1,745 bilhões de euros, um crescimento de $17 \%$ em relação ao ano anterior. As casas de apostas oferecem diversos mercados, como o mercado de odds fixas, no qual o apostador deve escolher se uma equipe ganha, perde ou empata; ou, ainda, mercados de under/over de gols, nos quais a entrada é selecionada pelo apostador como sendo uma quantidade abaixo ou acima de um valor fixo.

Construir modelos que tornem possível a predição de resultados é um desafio complexo. Isso devido às variáveis psicológicas, estratégicas, climáticas, físicas e locais que atuam diretamente sobre o resultado. Essas características, são, ainda, funções da qualidade individual dos jogadores e da comissão técnica. Além disso, as partidas dependem das tomadas de decisão coletiva e individual, ademais, podem sofrer interferência das decisões dos juízes da partida. Mascarenhas et al. (2006) realizaram um experimento com juízes profissionais, no qual 200 situações reais de jogo, previamente classificadas, foram novamente rotuladas pelos profissionais. No experimento, foi obtido um erro aproximado de $20 \%$ nas classificações feitas pelos profissionais em relação às classes reais.

Sob esse contexto, diversos modelos foram construídos ao longo do tempo. Essas abordagens podem ser classificadas em dois grandes grupos: (i) Que utilizam odds das casas de apostas e estatísticas dos times, com o objetivo de prever a equipe ganhadora da partida; e (ii) abordagens que empregam regressões lineares com objetivo de encontrar o número de gols da partida, ou quaisquer outro evento.

Moroney (1962) faz uma análise mais superficial, utilizando a Distribuição de Poisson, construindo um modelo que descreve o comportamento da quantidade de gols marcados por partida.

Reep e Benjamin (1968) realizam uma análise também baseada em distribuições binomiais, com um dataset composto por dados históricos de passes certos e chances de gols das equipes. Concluem, então, uma correlação entre a qualidade dos passes certos e os resultados dos jogos.

Clarke e Norman (1995) constroem um modelo baseado nas características de jogo de cada time. Esse modelo denota a Home Advantage, que é uma característica individual de cada time, calculada por uma relação de atributos 
estatísticos dos times o os seus respectivos desempenhos nos campeonatos jogados. Concluem, ainda, que, ao categorizar os dados por temporadas/anos, os resultados sofrem uma influência considerável.

Huang e Chang (2010) utilizam redes neurais alimentadas com dados da Copa do Mundo de 2006. Nessa abordagem, realiza-se uma normalização relativa dos dados para treinar uma modelo de perceptron multicamadas e dados os experimentos e obtém-se 76,9\% de precisão. Os atributos escolhidos para treinamento são os eventos ocorridos durante as partidas, como posse de bola, chutes a gol, etc. Já para o teste, realiza-se uma inferência com dados médios relativos aos três últimos jogos dos times.

Hucaljuk e Rakipović (2011) utilizam características como: os resultados dos últimos jogos, posição na tabela do campeonato, número de gols marcados e resultados de jogos fora de casa. Além disso, o modelo é construído para selecionar e comparar diferentes algoritmos, como: Naive Beyes, Bayesian Networks, Logistic Boost, K-Neighbors, Random Forest e Artificial Neural Networks. A abordagem apresentou acuracidade de $68 \%$.

Prasetio et al. (2016) propuseram um modelo baseado em técnicas de Regressões Lineares, realizando um estudo sobre as principais características utilizadas por pesquisadores no nicho, assim, buscando encontrar correlações entre essas características.

Srinivasan (2017) propõe uma nova técnica para predizer resultados de futebol baseados em dados estatísticos compostos por uma quantização dos passes até a realização de um determinado evento no decorrer do jogo.

Neste trabalho, é proposto um modelo de predição de resultados de futebol, utilizando dados históricos da Premier League e Bundesliga, e, valendo-se de características históricas dos últimos jogos das equipes, constrói-se um modelo classificatório baseado em técnicas de aprendizagem de máquinas.

O modelo visa prever qual equipe (mandante ou visitante) possui maior chances de vitória, baseado nos dados coletados. Para isso, utiliza-se o algoritmo Máquinas de Vetores de Suporte, ou em inglês, Support Vector Machines (SVM), que são modelos de classificação baseados em um treinamento supervisionado. Além disso, compara-se os melhores resultados dos modelos SVM com outros algoritmos de classificação. Ainda, realiza-se um comparação com outras pequisas e seus modelos propostos.

O trabalho é organizado nas seguintes seções: na Seção 2, é formalizado o conceito de SVM; na Seção 3, define-se os parâmetros de otimização; já na Seção 4 são explicados os métodos experimentais utilizados; e, por fim, a Seção 6 que trata das conclusões.

\section{MÁQUINAS DE VETORES DE SUPORTE}

As SVM são modelos de geração dicotômica, a partir de um treinamento supervisionado. A partir de um conjunto de dados $\left(\mathbf{x}_{i}, y_{i}\right)$ em que $\mathbf{x}_{i}$ representa um elemento do conjunto $\mathbf{X}$ de exemplos e $y_{i}$ seu rótulo.

Então, é construído um modelo classificador que seja capaz de prever um rótulo para um novo elemento $\mathbf{x}_{n}$, até então desconhecido. No modelo de aprendizado supervisionado, a presença do elemento $y_{i}$, denominado rótulo, tem como função inserir no modelo o conhecimento do ambiente externo (conjunto de dados de treino, $X$ ).

Existem duas possíveis abordagens nesse modelo: os problemas de classificação, em que as classes (ou rótulos) fazem parte de um conjunto de valores discretos $(0,1,2, \ldots, n)$, no qual, se $n=2$, o problema é binário e se $n>2$, a abordagem é denominada multi classes.

Cada amostra $\mathbf{x}_{i}$ do conjunto de treinamento $\mathbf{X}$ é representado por vetor de características que determinam um aspecto da amostra. Essas características podem ser categóricas ou contínuas. As categóricas determinam um grupo a essa característica, como, por exemplo: cores, local, campeonato, ano e etc. Já as características contínuas definem uma ordem linear aos valores, como, por exemplo: número de gols, escanteios, posse de bola. Neste trabalho, são abordados métodos de classificação binária.

$\mathrm{Na}$ sua forma mais simples, as SVM são hiperplanos que separam dados de acordo com uma margem. Todos os vetores alocados em um dos lados do hiperplano, são rotulados como -1 , e, os que repousam ao lado oposto ao hiperplano, são classificados como 1. Os vetores que definem o hiperplano são os vetores de suporte (Tong e Koller, 2001).

Seja $\Phi: \mathbf{R}^{N} \rightarrow F$ um mapeamento, onde $\mathbf{R}$ é o espaço de entrada de dimensão $N$ e $F$ é o espaço de características. Agora, seja a função $k$ denominada de kernel, definida por (1).

$$
k\left(\mathbf{x}_{i}, \mathbf{x}_{j}\right)=\Phi\left(\mathbf{x}_{i}\right) \cdot \Phi\left(\mathbf{x}_{j}\right) .
$$

Os kernels avaliados neste trabalho são: o polinomial (2), o RBF (Radial-Basis Function) (3) e os Sigmodais (4).

$$
\begin{gathered}
k\left(\mathbf{x}_{i}, y_{i}\right)=\left(\delta\left(\mathbf{x}_{i} \cdot \mathbf{x}_{j}\right)+\kappa\right)^{d}, \\
k\left(\mathbf{x}_{i}, y_{i}\right)=\exp \left(-\sigma\left\|\mathbf{x}_{i}-\mathbf{x}_{j}\right\|^{2}\right), \\
k\left(\mathbf{x}_{i}, y_{i}\right)=\tanh \left(\delta\left(\mathbf{x}_{i} \cdot \mathbf{x}_{j}\right)+\kappa\right) .
\end{gathered}
$$

Cada kernel depende de parâmetros determinados pelo usuário $(\delta, \kappa, \sigma$ e $d)$ e observa-se também que o kernel polinomial torna-se linear quando $d=1$. A formulação não linear adapta-se a melhor a situações reais devido a natureza dos dados, como outliers e ruídos.

\section{PARÂMETROS DE OTIMIZAÇÃO}

A otimização de uma SVM, formulada por (6), como uma função restrita ao termo de regularização w e ao erro individual de cada evento do treino $\xi$, sujeito a

$$
y_{i}\left(\mathbf{w}^{T} \mathbf{x}_{i}+b\right) \geq 1-\xi_{i},
$$

onde $i=1, \ldots, N$, sabendo que $\mathbf{w} \in \mathbb{R}^{d}$ e $\xi_{i} \in \mathbb{R}^{+}$.

$$
\min _{w, \xi}\|\mathbf{w}\|^{2}+C \sum_{i=1}^{m} \xi_{i} .
$$

A restrição $y_{i}\left(\mathbf{w}^{T} \mathbf{x}_{i}+b\right) \geq 1-\xi_{i}$, pode ser reescrita como

$$
\left.y_{i} f\left(\mathbf{x}_{i}\right)\right) \geq 1-\xi_{i} \text {. }
$$

Então, sabendo que $\xi_{i} \geq 0$, escreve-se a equivalência

$$
\xi_{i}=\max \left(0,1-y_{i} f\left(\mathbf{x}_{i}\right)\right)
$$


logo, reescrevendo, temos que

$$
\min _{w, \xi}\|\mathbf{w}\|^{2}+C \sum_{i=1}^{N} \max \left(0,1-y_{i} f\left(\mathbf{x}_{i}\right)\right) .
$$

A função de decisão é dada por (10), onde $\alpha_{i}$ é o multiplicador de Lagrange $(\mathrm{i}=1, \ldots, \mathrm{N})$.

$$
f(x)=\operatorname{sng}\left(\sum_{i=1}^{N} y_{i} \alpha_{i}^{*} k\left(\mathbf{x}_{i}, \mathbf{x}_{j}\right)+b\right) .
$$

Dessa forma, categoriza-se os pontos em três grupos:

- $y_{i} f\left(\mathbf{x}_{i}\right)>1$ : Ponto fora da margem, nenhuma contribuição para função de erro.

- $y_{i} f\left(\mathbf{x}_{i}\right)=1$ : Também nenhuma contribuição para função, é um caso de margem rígida.

- $y_{i} f\left(\mathbf{x}_{i}\right)<1$ : Ponto fora da margem, há uma contribuição à função de erro.

Valores grandes de $\mathrm{C}$ aumentam a penalidade por erros de classificação nos dados de treinamento. Isso corresponde a margens mais estreitas. Valores grandes de C também correspondem a menos vetores de suporte, isso porque menos observações são classificadas incorretamente e também menos observações estão dentro das margens mais estreitas (Guenther e Schonlau, 2016).

\section{METODOLOGIA}

O banco de dados deste trabalho é composto por 236 jogos da temporada 18/19 da Premier League (PL), Inglaterra somados a 164 partidas da Bundesliga 18/19 (BL), Alemanha. Os dados foram obtidos através de um crawler desenvolvido exclusivamente para este trabalho. O script busca uma série histórica de informações das partidas no site resultados.com e calcula uma média de cada característica em relação aos últimos cinco jogos de cada equipe. A Tabela 1 expõe a divisão dos dados em treino e teste.

Tabela 1. Divisão dos dados em treino e teste

\begin{tabular}{ccccc}
\hline \multicolumn{2}{c}{ Dataset } & Treino & Teste & Total \\
\hline \multirow{2}{*}{ PL } & Home & $95(67,37 \%)$ & $46(32,63 \%)$ & 141 \\
& Away & $62(65,26 \%)$ & $33(34,74 \%)$ & 95 \\
\hline \multirow{2}{*}{ BL } & Home & $60(61,85 \%)$ & $37(38,15 \%)$ & 97 \\
& Away & $51(76,11 \%)$ & $16(23,89 \%)$ & 67 \\
\hline
\end{tabular}

As características (e seus acrônimos) extraídas por jogo para cada time são: odds antes do início da partida (ODD), posse de bola (BP), tentativas ao gol adversário (ATT), finalizações (FIN), chutes para fora (FO), faltas cobradas (FC), escanteios a favor (CK), impedimentos (IMP), defesas do goleiro (GKD), total de passes acertados (PASS), vitórias (WIN), empates (DRAW), derrotas (LOSS), vítorias em confrontos diretos (MW) e média de gols a favor (SCO).

Os jogos recebem uma classificação binária de acordo com o vencedor da partida: (Home, 0) - time mandante foi o vitorioso e $($ Away, 1) - time visitante foi o ganhador.

\subsection{Pré-processamento}

A normalização do dataset deste trabalho é uma variação do método utilizado por Huang e Chang (2010). Mas, para a abordagem aqui proposta, calcula-se exclusivamente os dados da equipe mandante em relação à visitante. $\mathrm{O}$ algoritmo de normalização proposto pode ser visto em (11).

$$
f_{i}=\left\{\begin{array}{ll}
0,5, & c_{i a}=c_{i b} \\
\frac{c_{i a}}{c_{i a}+c_{i b}}, & c_{i a} \neq c_{i b}
\end{array},\right.
$$

onde i varia de 1-15. Então, por (11), as características $c_{1}, \ldots, c_{15}$ são convertidas para $f_{1}, \ldots, f_{15}$. Os subscritos ( $a$ e $b$ ) fazem referência aos times mandantes (Home) e aos visitantes (Away), respectivamente. Para exemplificar a estratégia utilizada, a Tabela 2 aponta a normalização das características da partida entre Everton e Chelsea realizada pela $\mathrm{PL}$, tendo como vitoriosa a equipe mandante (Everton), pelo placar de 2 a 0 .

Tabela 2. Normalização de dados, partida entre Everton vs. Chelsea (2-0)

\begin{tabular}{ccccc}
\hline \multirow{2}{*}{ Acrônimo } & \multirow{2}{*}{ Característica } & \multicolumn{2}{c}{ Entrada } & Saída \\
\cline { 3 - 5 } & & Home & Away & $f_{i}$ \\
\hline ODD & $c_{1}$ & 4,6 & 1,83 & 0,715 \\
\hline BP & $c_{2}$ & 40,33 & 42 & 0,489 \\
\hline ATT & $c_{3}$ & 10,67 & 6,33 & 0,627 \\
\hline FIN & $c_{4}$ & 3,33 & 3 & 0,526 \\
\hline FO & $c_{5}$ & 5 & 2,33 & 0,682 \\
\hline FC & $c_{6}$ & 12,33 & 14,33 & 0,462 \\
\hline CK & $c_{7}$ & 4,67 & 3 & 0,608 \\
\hline IMP & $c_{8}$ & 2,33 & 2,33 & 0,500 \\
\hline GKD & $c_{9}$ & 1,67 & 4 & 0,294 \\
\hline PASS & $c_{10}$ & 320,33 & 453,67 & 0,413 \\
\hline WIN & $c_{11}$ & 1 & 3 & 0,250 \\
\hline DRAW & $c_{12}$ & 1 & 1 & 0,500 \\
\hline LOSS & $c_{13}$ & 3 & 1 & 0,750 \\
\hline MW & $c_{14}$ & 1 & 4 & 0,200 \\
\hline SCO & $c_{15}$ & 1 & 2 & 0,334 \\
\hline & & & &
\end{tabular}

Para uma análise do dataset, torna-se necessário definir o conceito de odds: é a probabilidade estimada de um evento acontecer, devido a uma estratégia da casa de aposta. Por exemplo, para o jogo entre Arsenal e Manchester United, realizado no dia 10/03/2019, as odds disponíveis na casa de aposta da Bet365 eram de 2,37 para vitória do Arsenal, 3,60 para um empate e de 3,10 em caso de aposta na vitória do Manchester United.

Em posse desses valores, torna-se possível obter as probabilidades estimadas através da relação $p_{i}=1 /$ odd $_{i}$. Assim, obtemos, $42,2 \%$ de chances para o Arsenal, 27, $8 \%$ para o empate e de $32,3 \%$ para a equipe visitante. O somatório dessas probabilidades oferecidas na casa de aposta é 102, $3 \%$. O valor excedente, que, neste exemplo, é de $2,3 \%$ é o lucro esperado da casa de aposta.

Verifica-se, na Figura 1, um gráfico que relaciona (ODD) com a característica (LOSS). Observa-se, então, uma relação linearmente dependente entre as variáveis: com o aumento do valor relativo das derrotas (LOSS), a característica (ODD) também aumenta. A Figura 2, é composta pelo mapa de correlação entre o conjunto de características. 


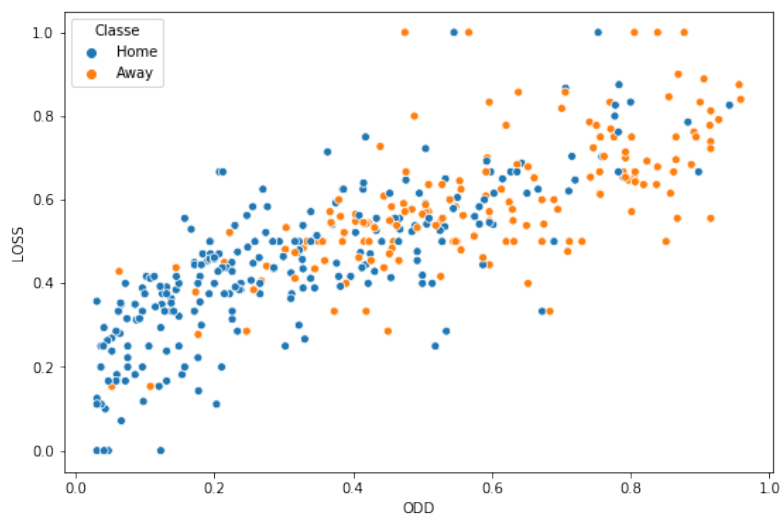

Figura 1. Relação entre características: ODD vs. LOSS.

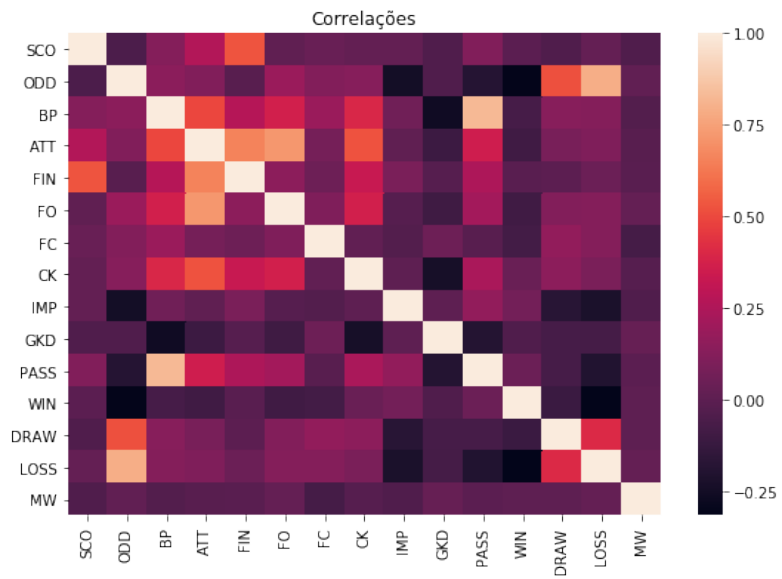

Figura 2. Correlação entre as variáveis do dataset.

\subsection{Avaliação dos modelos}

A acuracidade do modelo é definida como a razão entre a quantidade de amostras classificadas corretamente sobre o número de amostras classificadas. Erro quadrático Médio, ou Mean Square Error (MSE) é definido como uma função de risco correspondente ao valor esperado da perda do erro quadrático. Essa diferença é construída devido à aleatoriedade presente nos dados ou simplesmente à não habilidade do classificador em descrever as informações que poderiam fornecer uma estimativa mais precisa.

Neste trabalho, foram construídos três modelos - Kernel Polinomial (poly), Kernel Radial-Basis Function (RBF) e Kernel Sigmoidal (Sigmoid). Para cada modelo, foi explorada uma variação do parâmetro de regularização, com objetivo de encontrar o valor de $\mathrm{C}$ que maximalize o valor da acurácia, e, para cada valor de $\mathrm{C}$, o modelo foi iterado 10 vezes, depois obteve-se a média das métricas dessas iterações.

\section{RESULTADOS}

A Figura 3 demonstra o desempenho do kernel poly. A máxima acurácia obtida foi de $75,76 \%$ para um $C=$ 1 , nesse ponto, obteve-se $M S E=24,24 \%$. Observa-se também um decaimento da acurácia com o aumento da magnitude do parâmetro de regularização. O grau do polinômio $d$ em (2) é 2 .

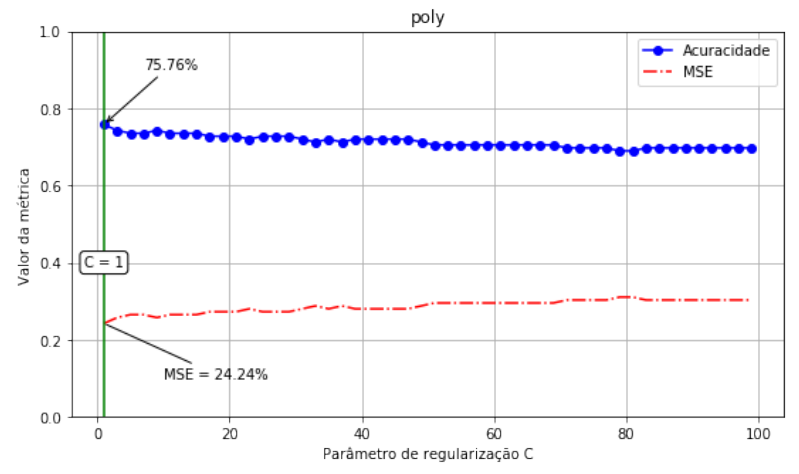

Figura 3. Kernel Polinomial.

Na Figura 4, observa-se a atenuação do valores da acurácia com o aumento do parâmetro de regularização no kernel RBF. O valor ótimo da acurácia $73,48 \%$, foi obtido para $C=1$ com um $M S E=26,52 \%$.

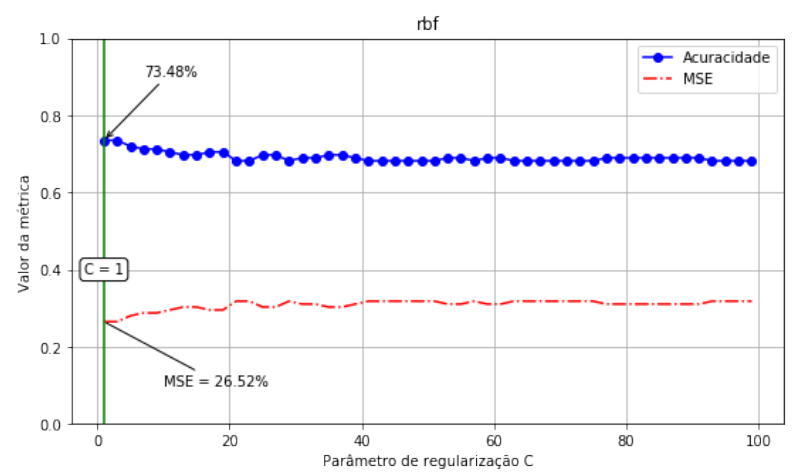

Figura 4. Kernel RBF.

A acurácia do Kernel Sigmoidal, Figura (5), possui baixa variação com o aumento de $C$, e obteve-se uma acurácia máxima de $74,24 \%$ para um $C=21$ com um $M S E=$ $25,76 \%$.

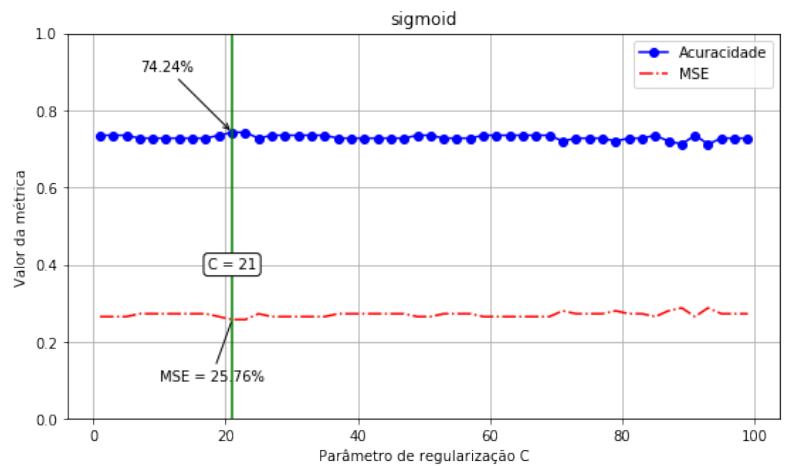

Figura 5. Kernel Sigmoidal.

Os desempenhos dos modelos está exposto na Tabela 3. Percebe-se uma baixa variação da acuracidade, mesmo com a variação dos hiperparâmetros do modelo. O melhor desempenho foi obtido pelo kernel poly com 75,76\% de acurácia e MSE, 24, 24\%, com um tempo de processamento médio de $0.03 \mathrm{~s}$. 
Tabela 3. Resultados de desempenhos dos Kernels.

\begin{tabular}{ccccc}
\hline Kernel & Acurácia & C & MSE & Tempo \\
\hline Poly & $75,76 \%$ & 1 & $24,24 \%$ & $0,03 \mathrm{~s}$ \\
RBF & $73,48 \%$ & 1 & $26,52 \%$ & $0,02 \mathrm{~s}$ \\
Sigmoid & $74,24 \%$ & 21 & $25,76 \%$ & $0,31 \mathrm{~s}$ \\
\hline
\end{tabular}

A Figura 6 denota as fronteiras de decisão de cada kernel com os parâmetros ótimos obtidos nos testes empíricos, as características plotadas são (ODD) e (LOSS).
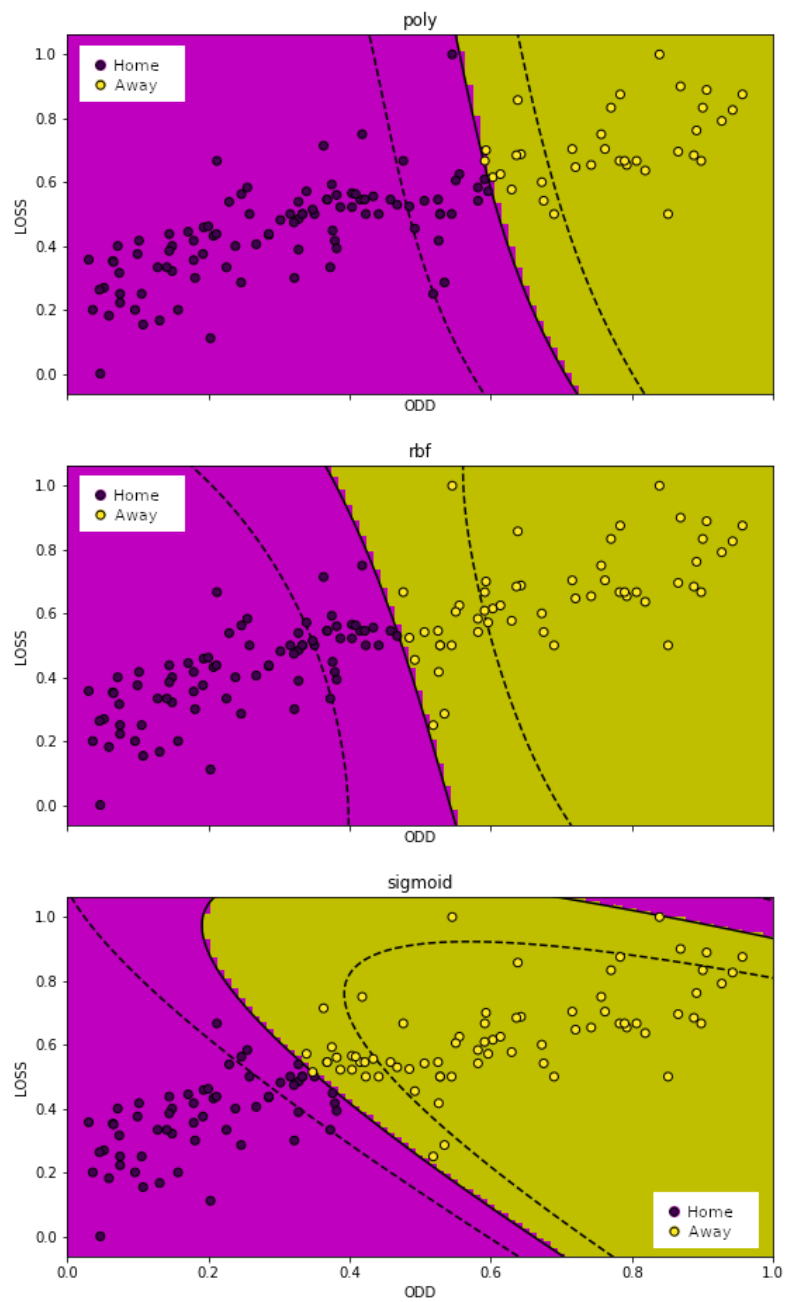

Figura 6. Fronteiras de decisão para os dados de teste.

Então, para verificar a qualidade do modelo SVM, procurase a comparação com outros classificadores (Clf). Na Tabela 4, verifica-se o melhor desempenho obtido pelos classificadores SVM (kernel polinomial, $C=1$ ) comparado a outros dois algoritmos de aprendizado supervisionado: Perceptron Multicamadas (MPL) e KNeighbors (KNN).

Tabela 4. Resultados de desempenho de diferentes classificadores.

\begin{tabular}{clclc}
\hline Clf. & Acc. & Parâmetro & MSE & Tempo \\
\hline SVM & $75,76 \%$ & C=1 & $24.24 \%$ & $0,03 \mathrm{~s}$ \\
MLP & $72,16 \%$ & hidden_layer_sizes $=15$ & $27,84 \%$ & $3,50 \mathrm{~s}$ \\
KNN & $68,94 \%$ & n_neighbors $=27$ & $31,06 \%$ & $0,05 \mathrm{~s}$ \\
\hline
\end{tabular}

O modelo MLP foi iterado 20 vezes e apresentou uma acurácia de $72,16 \%$, com o parâmetro hidden_layer_sizes= 15, que representa o número de neurônios na camada ocultada da rede. Ainda, obteve um $M S E=27,84 \%$ e um tempo médio de $3.50 \mathrm{~s}$.

O modelo KNN, também iterado 20 vezes, obteve uma acurácia média de 68,94\% com um $M S E=31,06 \%$ e tempo médio de execução de $0.05 \mathrm{~s}$.

\subsection{Outras pesquisas}

Comparar outros trabalhos de pesquisa não é simples, porque todos eles foram treinados com diferentes conjuntos de dados, características e métricas. Entretanto, considerando apenas a acuracidade como elemento comparativo, pode-se verificar na Tabela 5 um paralelo a pesquisas relacionadas.

Além do já citado MLP, os trabalhos utilizam os seguintes métodos: Long Short-Term Memory (LSTM), Artificial Neural Networks (ANN), Linear Regression (LR), Multinomial Regression e Random Forests (RF). Além disso os datasets utilizados contam com diferentes campeonatos como World Cup (WC), Champions League (CL) e a já citada Premier League (PL).

Tabela 5. Resultados de trabalho relacionados

\begin{tabular}{cccc}
\hline Pesquisa & Método & Dataset & Acc. \\
\hline (Danisik et al., 2018) & LSTM & PL, 15/16 & $52,5 \%$ \\
(Huang e Chang, 2010) & MLP & WC, 2006 & $76,9 \%$ \\
(Hucaljuk e Rakipović, 2011) & ANN & CL, 1996 & $68,0 \%$ \\
(Prasetio et al., 2016) & LR & PL, 15/16 & $69,5 \%$ \\
(Snyder, 2013) & MR & PL, 11/12 & $51,1 \%$ \\
\hline
\end{tabular}

Dentro os métodos analisados, Huang e Chang (2010) apresentam a maior acurácia, 76, 9\% Snyder (2013) apresenta a menor, com 51,1\%. Apesar de utilizar os mesmos algoritmos classificadores, os modelos apresentam divergências devido à natureza da construção. O modelo proposto neste trabalho performa-se inferiormente somente a Huang e Chang (2010), entretanto, os autores utilizam um dataset composto por um campeonato de natureza diferente dos demais (Copa), assim como Hucaljuk e Rakipović (2011). Dentro as pesquisas que utilizam campeonatos de pontos corridos (Premier League e Bundesliga) o método proposto neste trabalho obtém um desempenho superior aos modelos analisados. 


\section{CONCLUSÕES}

Este trabalho exibe experimentos e comparações entre modelos de aprendizagem supervisionada, com objetivo de predizer resultados de futebol. Nos experimentos, observou-se que um modelo SVM construído com um kernel polinomial de grau 2, com um parâmetro de regularização $C=1$, obtém melhores desempenhos ao ser submetido ao dataset proposto, $75,76 \% 5$. Em comparação com outros algoritmos de classificação supervisionada (MLP, 72, 16\%) e (KNN, 68,94\%), observou-se que o modelo proposto possui desempenho superior e menor custo computacional.

\section{AGRADECIMENTOS}

Os autores agradecem ao curso de Engenharia Elétrica da Universidade Federal do Piauí e ao Grupo de Pesquisa em Automação e Sistemas Inteligentes.

\section{REFERÊNCIAS}

Betfair, P.P. (2018). Annual report and accounts. 7.

Clarke, S.R. e Norman, J.M. (1995). Home ground advantage of individual clubs in english soccer. Journal of the Royal Statistical Society: Series D (The Statistician), 44(4), 509-521.

Danisik, N., Lacko, P., e Farkas, M. (2018). Football match prediction using players attributes. Em 2018 World Symposium on Digital Intelligence for Systems and Machines (DISA), 201-206. IEEE.

Guenther, N. e Schonlau, M. (2016). Support vector machines. The Stata Journal, 16(4), 917-937.

Huang, K.Y. e Chang, W.L. (2010). A neural network method for prediction of 2006 world cup football game. Em The 2010 international joint conference on neural networks (IJCNN), 1-8. IEEE.

Hucaljuk, J. e Rakipović, A. (2011). Predicting football scores using machine learning techniques. Em 2011 Proceedings of the 34th International Convention MIPRO, 1623-1627.

Mascarenhas, D., O Hare, D., Plessner, H., et al. (2006). The psychological and performance demands of association football refereeing. International Journal of Sport Psychology, 37(2/3), 99.

Moroney, M.J. (1962). Facts from figures. Penguin books.

Prasetio, D. et al. (2016). Predicting football match results with logistic regression. Em 2016 International Conference On Advanced Informatics: Concepts, Theory And Application (ICAICTA), 1-5. IEEE.

Reep, C. e Benjamin, B. (1968). Skill and chance in association football. Journal of the Royal Statistical Society. Series A (General), 131(4), 581-585.

Snyder, J.A.L. (2013). What actually wins soccer matches: Prediction of the 2011-2012 premier league for fun and profit. Master's thesis, Princeton University, NJ.

Srinivasan, B. (2017). A social network analysis of footballevaluating player and team performance. Em 2017 Ninth International Conference on Advanced Computing (ICoAC), 242-246. IEEE.

Tong, S. e Koller, D. (2001). Support vector machine active learning with applications to text classification. Journal of machine learning research, 2(Nov), 45-66. 